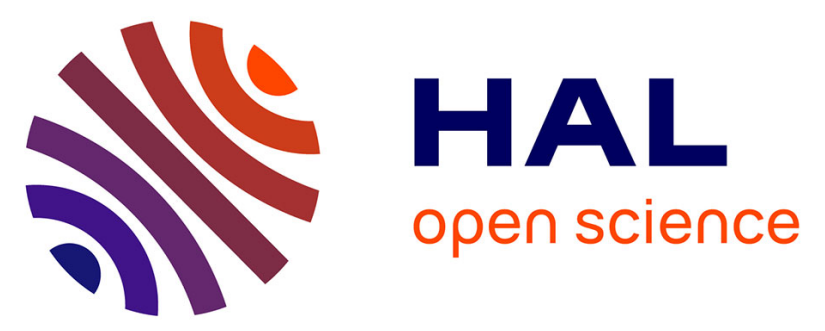

\title{
Structural insights into glutathione-protected gold Au10-12(SG)10-12 nanoclusters revealed by ion mobility mass spectrometry
}

Clothilde Comby-Zerbino, Franck Bertorelle, Fabien Chirot, Philippe Dugourd, Rodolphe Antoine

\section{To cite this version:}

Clothilde Comby-Zerbino, Franck Bertorelle, Fabien Chirot, Philippe Dugourd, Rodolphe Antoine. Structural insights into glutathione-protected gold Au10-12(SG)10-12 nanoclusters revealed by ion mobility mass spectrometry. The European Physical Journal D: Atomic, molecular, optical and plasma physics, 2018, 72 (8), pp.Article Number: 144. 10.1140/epjd/e2018-90133-8 . hal-01900250

\author{
HAL Id: hal-01900250 \\ https://hal.science/hal-01900250
}

Submitted on 31 Oct 2020

HAL is a multi-disciplinary open access archive for the deposit and dissemination of scientific research documents, whether they are published or not. The documents may come from teaching and research institutions in France or abroad, or from public or private research centers.
L'archive ouverte pluridisciplinaire HAL, est destinée au dépôt et à la diffusion de documents scientifiques de niveau recherche, publiés ou non, émanant des établissements d'enseignement et de recherche français ou étrangers, des laboratoires publics ou privés. 


\title{
Structural Insights into Glutathione-Protected Gold $\mathrm{Au}_{10-12}(\mathrm{SG})_{10-12}$ Nanoclusters Revealed by Ion Mobility Mass Spectrometry
}

\author{
Clothilde Comby-Zerbino ${ }^{1}$, Franck Bertorelle ${ }^{1}$, Fabien Chirot $^{2}$, Philippe Dugourd ${ }^{1}$ and Rodolphe \\ Antoine ${ }^{1 *}$ \\ ${ }^{1}$ Institut lumière matière, UMR5306 Université Claude Bernard Lyon1-CNRS, Université de Lyon 69622 Villeurbanne \\ cedex, France. \\ 2 Univ Lyon, CNRS, Université Claude Bernard Lyon 1, ENS de Lyon, Institut des Sciences Analytiques, UMR 5280,5 \\ rue de la Doua, F-69100 VILLEURBANNE, France.
}

\section{An invited contribution to}

EPJ D Special issue: Atomic Cluster Collisions

https://epjd.epj.org/open-calls-for-papers/100-epj-d/1382-epjd-special-issue-atomic-cluster-collisions

\begin{abstract}
:
Gold nanoclusters protected by ligands present well-defined compositions and tunable structures, which builds a solid basis for correlation between structures and properties. We report a combined ion mobility-mass spectrometry approach for the analysis of ultra-small gold nanoclusters protected by glutathione (SG) as ligand molecules, $\mathrm{Au}_{10-12}(\mathrm{SG})_{10-12}$. Collision cross section (CCS) measurements are reported for different charge states for $\mathrm{Au}_{10}(\mathrm{SG})_{10}, \mathrm{Au}_{11}(\mathrm{SG})_{11}$ and $\mathrm{Au}_{12}(\mathrm{SG})_{12}$ nanoclusters. Computational calculations, at the PM7 semi-empirical level of theory, are performed to optimize geometrical structures and use them to compute CCS. The comparison of the experimentally and theoretically determined CCS allows drawing conclusions on the structural changes, in particular partial unfolding of SG ligands, upon charging.
\end{abstract}

\section{Introduction:}

Metal nanoclusters (NCs) consisting of several to a few hundred metal atoms and protected by organic ligands exhibit extraordinary physical and chemical properties. ${ }^{[1,2]}$ They display absorption and emission properties via electronic transitions. ${ }^{[3]}$ Their sizedependent fluorescence, good photostability and biocompatibility render them very attractive as potential biomedical probes. ${ }^{[4-6]}$ Since their physicochemical properties are highly size- and structure-dependent, a precise characterization of the composition and structure of those clusters at the atomic level is crucial towards the rationalization of their synthesis and the design of new clusters with tailored properties.

Mass spectrometry (MS) has shown considerable potential in this field for its capability to provide information on both metal core size and molecular composition. ${ }^{[7}$, 8] Ion mobility spectrometry (IMS) has been proposed as a useful complement to MS due to its sensitivity to $3 \mathrm{D}$ molecular structures. 
Thus this complementary technique has been recently proposed for the analysis of gasphase ligand-protected metal nanoclusters. IMS provides separation selectivity according to the ion size, specifically ion surface area. Briefly, in IMS, ions travel a drift tube filled with an inert gas. Ions experience many lowenergy collisions with the background gas which separates ions based on their ionneutral collision cross section (CCS). Following the pioneering IMS work of Bowers et al. ${ }^{[9]}$ and Jarrold et al. ${ }^{[10]}$ on metal cluster ions, in 2002, Kappes and colleagues determined the structures of small gold cluster cations ${ }^{[11]}$ and anions ${ }^{[12]}$ by a combination of ion mobility measurements and density functional calculations. This ability to distinguish between diverse 3D geometries is also appealing for ligand-protected gold clusters.

A significant breakthrough was achieved by Cliffel, McLean and colleagues who reported for the first time, a combined ion mobilitymass spectrometry (IM-MS) for the analysis of gold nanoclusters protected by tiopronin or phenylethanethiolate as well as goldtiopronin and gold-phenylethanethiolate precursor complexes. ${ }^{[13]}$ Right after, Dass et al. combined mass spectrometry (MS), collision-induced dissociation (CID) and ion mobility (IM), to characterize
$\mathrm{Au}_{25}\left(\mathrm{SCH}_{2} \mathrm{CH}_{2} \mathrm{Ph}\right)_{18}{ }^{-[14]}$ Then IM-MS was evaluated for its capabilities in separation of isomeric gold nanoclusters. Laskin et al. reported structural changes in gold clusters induced by interactions between differently substituted phosphine ligands and gold cores. $^{[15]}$ Also, the Pradeep group reported the first observation of the presence of ligand induced isomers of glutathione protected $\mathrm{Ag}_{11}$ by IM-MS where two isomers can be distinguished by their different drift times. ${ }^{[16,}$ ${ }^{17]}$ Our group showed how IM MS studies can get insight into the size ${ }^{[18]}$ of glutathioneprotected gold nanoclusters and can help in structural determination ${ }^{[19]}$ in the case of inorganic nanoclusters.

In a previous work, we reported a facile "onepot-one-size" synthesis of $A u_{10}(S G)_{10}$ NCs ((SG : glutathione : $\gamma$-L-Glutamyl-L-cysteinylglycine see Fig. 1b) characterized by electrospray MS. The distinct $X$-ray diffraction pattern of $\mathrm{Au}_{10}(\mathrm{SG})_{10}$ was utilized as a signature for homoleptic gold-glutathione catenanes (see Fig. 1a). ${ }^{[20]}$ In this work, we study a series of as-synthesized $\quad \mathrm{Au}_{10-12}(\mathrm{SG})_{10-12}$ glutathioneprotected gold nanoclusters with zero confined electrons (they do not have any metal core) using ion mobility spectrometry and mass spectrometry. 


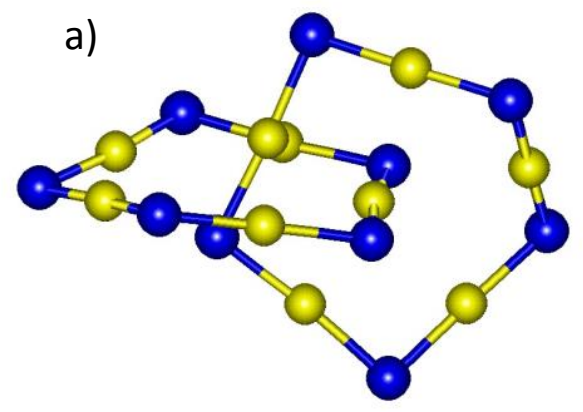

b)
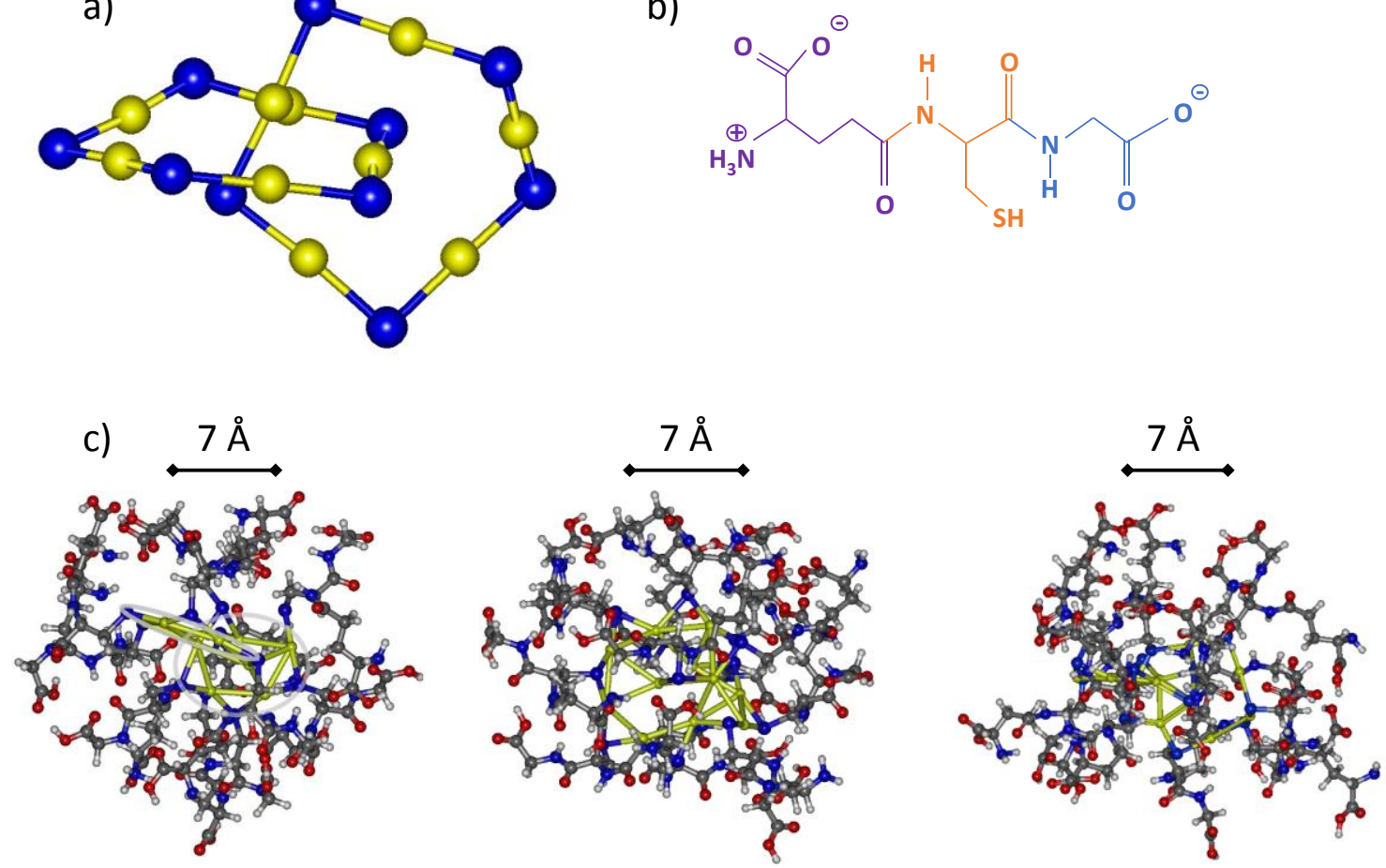

Calculated

$\operatorname{CCS}_{\text {Hе }}\left(\AA^{2}\right): \quad 495$

546

557

Figure 1: (a) schematic model of catenane structures : [5,5] (interconnected rings of $\left.A u_{5} S_{5}\right)$. (b) chemical structure of the glutathione tripeptide ligand : $y$-L-Glutamyl-L-cysteinylglycine. (c) PM7 optimized catenane-like structures for $A_{10} u_{10-12} S G_{10-}$ 12. Grey lines are drawn on the core structure of $A u_{10} S G_{10}$ to highlight the catenane structure. CCS values calculated, from the neutral form of catenane-like structures with the trajectory method, are also given.

SG ligand is a bulky and highly flexible peptide ligand, thus one the main issue is to evaluate the possibility to disentangle the importance of core structure and the ligand conformations on the total CCS. Indeed, unlike larger clusters (e.g. $\mathrm{Au}_{15}(\mathrm{SG})_{13}$, $\mathrm{Au}_{18}(\mathrm{SG})_{14}$, and $\left.\mathrm{Au}_{25}(\mathrm{SG})_{18}\right), \mathrm{Au}_{10-12}(\mathrm{SG})_{10-12}$ clusters display no metal core but rather interacting AuSG rings. Thus, for such smaller clusters, it is expected that the ligand conformations may have a more dramatic impact on their total collision cross section. For this purpose, computational calculations, at the PM7 semi-empirical level of calculation, of the catenane-like structure of the clusters have been performed, and used to obtain theoretical CCS-values. This combined approach is evaluated in order to gain detailed insight in the structural properties of $\mathrm{Au}_{10-12}(\mathrm{SG})_{10-12}$ clusters in terms of size, composition, and conformation. The comparison of the experimentally and theoretically determined CCS allows drawing conclusions on the sensitivity of IMS to resolve catenane-like structures and to evaluate the structural changes, in particular partial unfolding of SG ligands, upon charging.

\section{Materials and Methods}




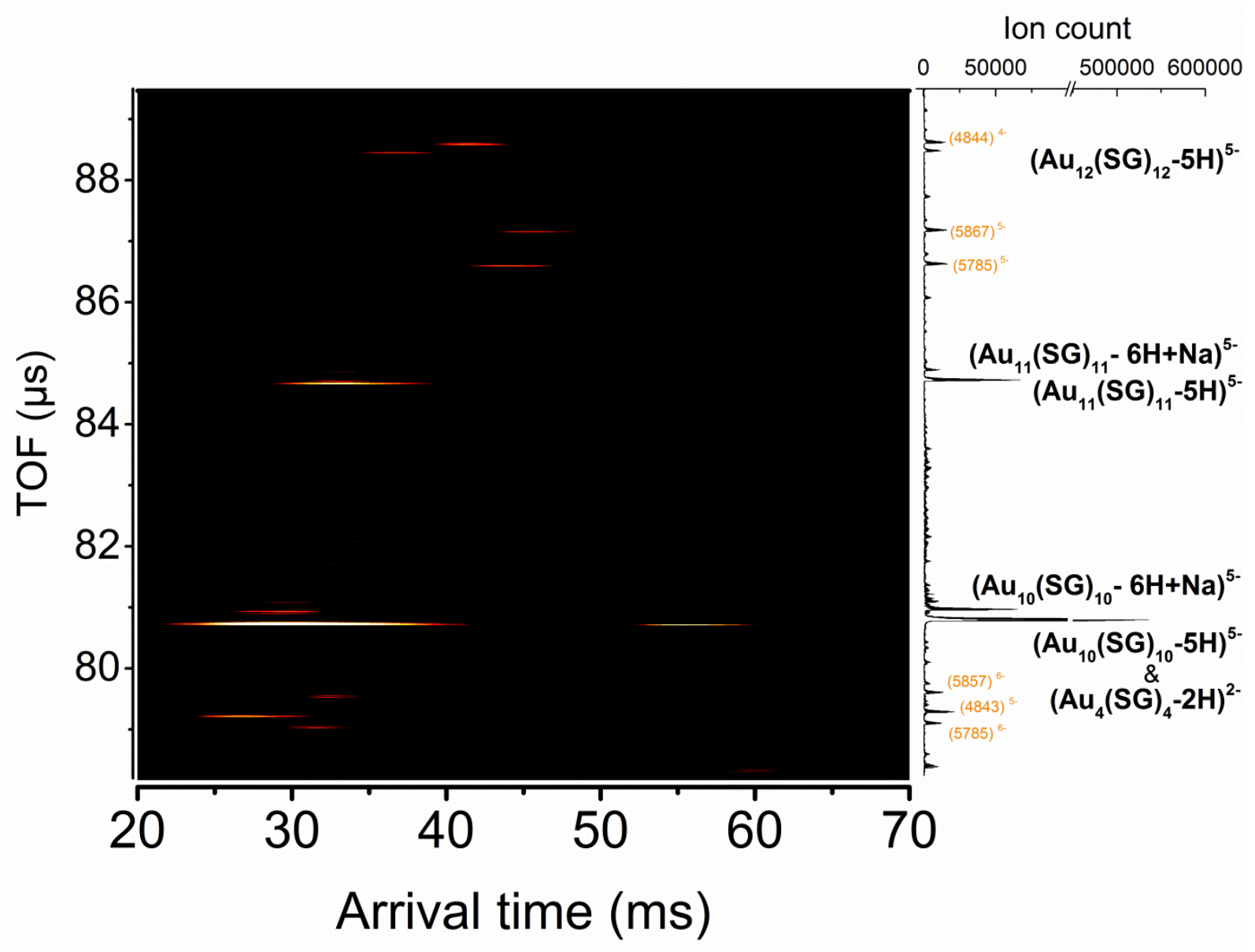

Figure 2: IM-MS map showing time of flight (y axis) versus arrival drift time (x axis) for the analysis of $\mathrm{Au}_{10-12}(\mathrm{SG})_{10-12 .}$ The color corresponds to an intensity scale from black (low) to white (high). The results were obtained using 4.15 Torr Helium as a buffer gas at $296 \mathrm{~K}$, with a $450 \mathrm{~V}$ drift voltage across the drift tube. $\left(\mathrm{Au} \mathrm{u}_{n}(\mathrm{SG})_{n}-\mathrm{pH}+\mathrm{Na}\right)^{\mathrm{x}-}$ correspond to sodiated cluster species.

$A u_{10-12} S_{10-12}$ Nanocluster synthesis. $A u_{10}$ ${ }_{12} \mathrm{SG}_{10-12} \mathrm{NCs}$ were prepared as follows: $235 \mathrm{mg}$ of glutathione is dissolved in $50 \mathrm{ml}$ of methanol and $2 \mathrm{ml}$ of triethylamine. $100 \mathrm{mg}$ of $\mathrm{HAuCl}_{4}$, $3 \mathrm{H}_{2} \mathrm{O}$ in $1 \mathrm{ml}$ of water is added and the solution is stirred

overnight at ambient temperature. To complete precipitation, $2 \mathrm{ml}$ of $1 \mathrm{M} \mathrm{NaOH}$ solution is added and the solution is centrifuged $(5 \mathrm{mn}$ at $9000 \mathrm{rpm})$. The unwanted products are removed with one cycle of dissolution/precipitation/centrifugation.
Mass spectrometry and ion-mobility. Ion mobility measurements were performed using a custom-built tandem ion mobility spectrometer described in detail elsewhere. ${ }^{[21]}$ The results below were obtained using a fresh mixture of $\mathrm{Au}_{10-12}(\mathrm{SG})_{10-}$ 12, prepared in aqueous solution to a concentration of around $50 \mu \mathrm{M}$. This solution was electrosprayed directly using a syringe pump. Mobility measurements were done by injecting short $(200 \mu \mathrm{s})$ ion bunches in a 79 $\mathrm{cm}$-long drift tube filled with 4.15 Torr helium, 
in which a constant drift field was maintained through the controlled voltage drop across the tube (in the 200-500 V range). The temperature of the whole instrument was kept at $296 \mathrm{~K}$. After their drift, ions were conveyed to a reflector time-of-flight(TOF) mass spectrometer. Mass spectra were finally recorded as a function of the IMS drift time, allowing extraction of arrival time distributions (ATDs) for ions with any desired mass-to-charge ratio. Such ATDs were recorded for different drift voltages in order to determine absolute values for the collision cross-sections (CCS) based on the linear relation between the drift time and the inverse drift field. Under the experimental conditions the ions travel across the drift tube (with a length $\mathrm{L}$ ) at a constant speed $v_{D}$, and their drift time $t_{D}$ through the tube is linked to this field through:

$t_{D}=\frac{L}{v_{D}}=\frac{L}{K E}$

where $K$ defines the ion mobility. $K$ is inversely proportional to the orientationally averaged diffusion cross-section CCS :[22]

$K=\frac{3}{16} \times \frac{z e}{N} \times\left(\frac{1}{m}+\frac{1}{M}\right)^{1 / 2} \times\left(\frac{2 \pi}{k T}\right)^{1 / 2} \times \frac{1}{\mathrm{CCS}}$

where ze is the charge of the ion, $N$ is the buffer gas density, $k$ is the Boltzmann constant, $T$ is the temperature, and $m$ and $M$ are the masses of the helium atom and the ion, respectively. ${ }^{[22,23]}$

Computational. Starting from model catenane structures : $[5,5]$ (double ring $\mathrm{Au}_{5} \mathrm{SG}_{5}$ (see Fig. 1a); $[6,5]$ double ring $\mathrm{Au}_{6} \mathrm{SG}_{6}-$ $\mathrm{Au}_{5} \mathrm{SG}_{5}$; and $[6,6]$ double ring $\left.\mathrm{Au}_{6} \mathrm{SG}_{6}\right)$. $\mathrm{Au}_{10}$ ${ }_{12}(\mathrm{SG})_{10-12} \mathrm{NCS}$ with neutral glutathione ligands were optimized using the PM7 semi-empirical Hamiltonian implemented in the MOPAC2016 code, ${ }^{[24,25]}$ found to give reasonably good agreement for calculated interaction parameters for gold-sulfur complexes. ${ }^{[26]}$

\section{Results and discussion}

In the IM-MS approach, gas phase NCs ions are dispersed in two dimensions (IM-MS map), specifically size-to-charge (by their arrival time : IM) and mass-to-charge (by their time-of-flight TOF : MS). The examination of the IM-MS map obtained for $\mathrm{Au}_{10-12}(\mathrm{SG})_{10-12}$ clusters (Figure 2) first provides information on the structural diversity of this cluster. The extracted arrival time distributions ATDs are mainly monomodal for the 5- charge state of $\mathrm{Au}_{10}$ ${ }_{12}(\mathrm{SG})_{10-12}$, indicating that the corresponding clusters present essentially a single structural type. Of note, the ATD observed ( $55 \mathrm{~ms}$ ) right after the ATD of the 5- charge state of $\mathrm{Au}_{10}(\mathrm{SG})_{10}$ ( $\left.\sim 30 \mathrm{~ms}\right)$ is due to the 2-charge state of $\mathrm{Au}_{4}(\mathrm{SG})_{4}$ (see Figure 2). Also a series of ion signals are observed, with spacings in mass of $22 \mathrm{Da}$, typical for an exchange between $\mathrm{Na}^{+}$and $\mathrm{H}^{+}$as counter ions in the negatively charged ionic clusters (see figure2).

As described in the experimental section, an absolute value for the CCS of the clusters can be obtained by measuring ATDs at different drift voltages. The experimental CCSs determined for different charge states for $\mathrm{Au}_{10}(\mathrm{SG})_{10}, \mathrm{Au}_{11}(\mathrm{SG})_{11}$ and $\mathrm{Au}_{12}(\mathrm{SG})_{12}$ nanoclusters are given in Table 1. For a given cluster, the higher the charge state, the larger the collision cross-section (see Fig. 3). This may be related to the fact that the ligand molecule is a tripeptide that partially unfolds upon charging in the gas phase, leading to an increase in the collision cross-section as already observed for larger AuSG NCs. ${ }^{[18]}$ 


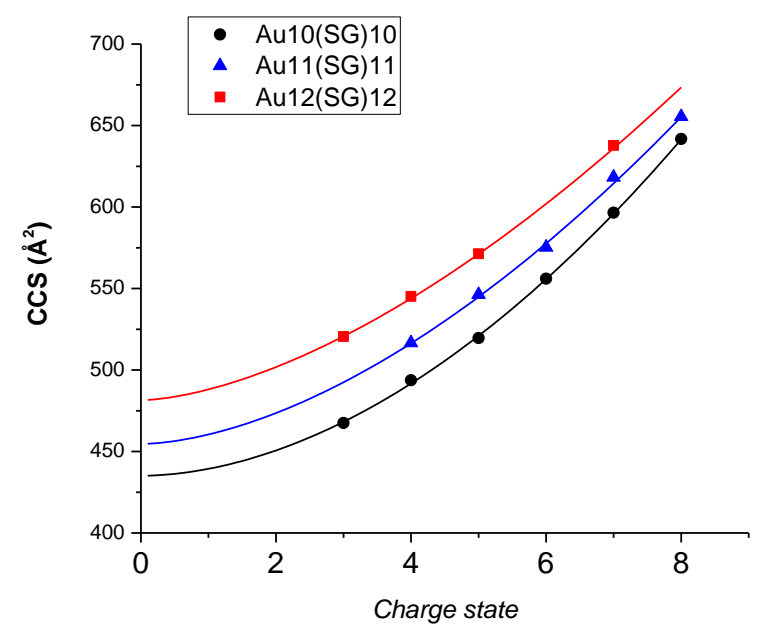

Figure 3: Collision cross-section measured in helium at 296 $K\left({ }^{D T C C S} \mathrm{He}_{\mathrm{e}}\right.$ for $A u_{10}(\mathrm{SG})_{10}, A u_{11}(S G)_{11}$ and $A u_{12}(S G)_{12} N C S$ as a function of their charge states in aqueous solution. Precision on CCS values is $+/-2 \%$. The continuous line matches to the allometric fit. The allometric function uses the relationship between two measured quantities (CCS and charge state) is expressed as a power law equation which expresses as : CCS $=k(\text { charge state })^{a}$.

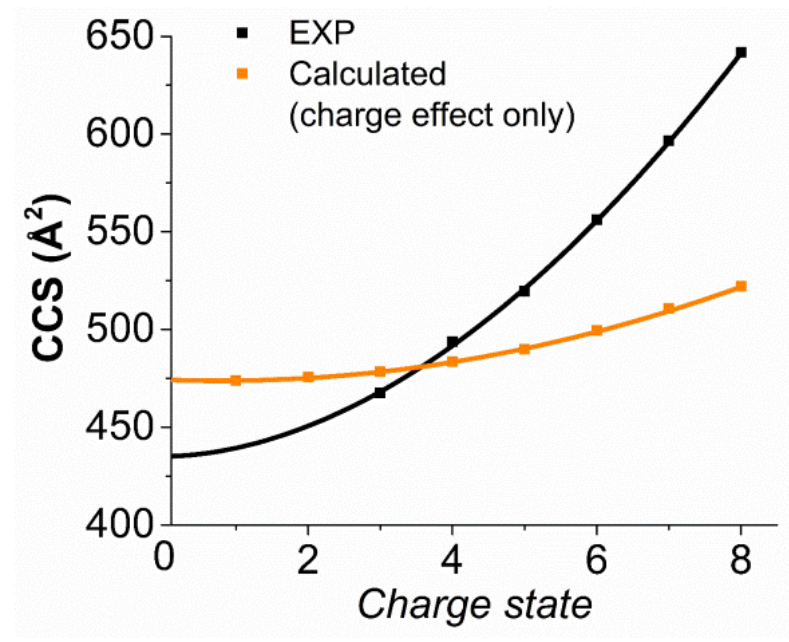

Figure 4: Experimental and calculated CCS values for different charge states of $A u_{10} S_{10}$ NC. The allometric function allowed an extrapolation of the ${ }^{D T} C C S_{H e}$ to charge state equal 0 , useful to compare with calculations. Calculated CCS calculated for a rigid catenane structure, assuming different charges for the gold core.

\begin{tabular}{|ccccccc|} 
& \multicolumn{7}{c|}{${ }^{\text {DTCCS }}$ ( $\left.\AA^{2}\right)$ vs Charge State } \\
\cline { 2 - 7 } Au NCs & $\mathbf{8 -}$ & $7-$ & $6-$ & $5-$ & $4-$ & 3- \\
\hline $\mathbf{A u}_{10}$ (SG) $)_{10}$ & 642 & 597 & 556 & 520 & 494 & 468 \\
\hline $\mathbf{A u}_{11}$ (SG) $)_{11}$ & 655 & 618 & 575 & 546 & 517 & \\
\hline $\mathbf{A u}_{12}$ (SG) & & 638 & & 571 & 545 & 510 \\
\hline
\end{tabular}

Table 1. Collision Cross-Section Measured in Helium at $296 K{ }^{(D T} C_{C C S}$ ) for Au NCs as a Function of Their Charge State. Precision on CCS Values $\pm 2 \%$

Optimized PM7 catenane-like structures (see Fig. 1c) were used for CCS calculations. The CCS values calculated with the IMOS trajectory method ${ }^{[27]}$ from the neutral form of catenanelike structures reasonably match the experimental data (in particular values for 4and 5- charge states). However, the resolving power of drift tube IMS does not seem sufficient to discriminate between different possible catenane structures, as illustrated in Fig. S1. Also, other geometrical structures may be in competition with catenane-like structures. As shown in our previous work, ${ }^{[20]}$ the crown-like structure can be a possible structure. Again, the resolving power of drift tube IMS does not seem sufficient to discriminate between crown-like and catenane structures. Another observation is that the calculated values are higher than the extrapolated ${ }^{\mathrm{DT}} \mathrm{CCS}_{\mathrm{He}}$ for charge state equal 0 using an allometric function (see Figs. 3 and 4). These differences between the calculated CCS and the experimental CCS value interpolated at a charge state equal 0 may originate from the complex packing of the glutathione ligands around the catenane structure. An exhaustive exploration of all the packing possibilities for the 10 to 12 glutathione ligands is out of the scope of the present work. However an important finding is the strong increase of CCSs vs charge state for all nanoclusters. The charge of an ion may affect its CCS for different reasons. On the one hand, ion-induced-dipole forces are responsible for part of the interaction between the ion and the buffer gas molecules, especially at long distances. ${ }^{[28]}$ For flexible 
molecules however, charge-induced unfolding due to Coulomb repulsion between charged moieties usually produces more dramatic effects on the CCS. ${ }^{[29]}$ This is especially true when the buffer gas is poorly polarizable, as helium. As an attempt to disentangle the share of the latter two effects in the present case, we computed CCS for a series of $\mathrm{Au}_{10} \mathrm{SG}_{10}$ catenane structures with fixed atomic coordinates, but bearing a different charge, evenly distributed on the atoms of the gold core. CCS were calculated using the Trajectory method implemented in the IMOS software, developed by LarribaAndaluz and Hogan, ${ }^{[27]}$ which takes electrostatic interactions into account. Clearly, at room temperature, the increase of CCS vs charge state cannot be explained only by the charge dependence of the ion-induced dipole interaction between the buffer gas and the ions (see Fig. 4). Thus this strong charge-state effect on experimental CCS values may originate from a charge induced glutathione unfolding.

\section{Conclusion}

In this work, we report a combined ion mobility-mass spectrometry (IM-MS) approach for the analysis of glutathioneprotected gold nanoclusters with zero confined electrons. CCS measurements are reported for different charge states for $\mathrm{Au}_{10}(\mathrm{SG})_{10}, \quad \mathrm{Au}_{11}(\mathrm{SG})_{11}$ and $\mathrm{Au}_{12}(\mathrm{SG})_{12}$ nanoclusters. CCS values are consistent with a catenane-like form for such nanoclusters, although the resolving power of drift tube IMS does not seem sufficient to discriminate between different possible catenane structures. However, strong charge-state effects on experimental CCS values are observed and may originate from a charge induced gluthatione unfolding. It is clear that with such bulky and highly flexible peptide ligand, it is difficult to disentangle the importance of core structure and the ligand conformations on the total CCS. Disentangling the share of the core and ligand to the observed conformational changes could by easier by using smaller/more rigid ligands. Work along these lines is currently underway in our laboratory.

\section{Supporting Information}

Catenane-like structures for $\mathrm{Au}_{10}(\mathrm{SG})_{10}$ composed by 2 interconnected $\mathrm{Au}_{5} \mathrm{SG}_{5}$ rings and 2 interconnected $\mathrm{Au}_{6} \mathrm{SG}_{6}$ and $\mathrm{Au}_{4} \mathrm{SG}_{4}$ rings.

\section{Acknowledgments}

The research leading to these results was funded by the European Research Council under the Seventh Framework Program of the European Union (FP7 Grant Agreement 20072013 No 320659).

\section{Author contribution statement}

RA conceived the initial idea and coordinated the work. FB synthesized and prepared the nanoclusters. CCZ measured CCS and recorded mass spectra. CCZ and FC analyzed the results. RA and PD supervised and financed the project. RA and FC wrote the paper. All the authors provided critical feedback and helped to shape the final manuscript.

\section{Corresponding Author}

\footnotetext{
*Rodolphe Antoine : Address: Institut lumière matière, UMR5306 Université Claude Bernard Lyon1-CNRS, 5 Rue de la Doua, 69100 Villeurbanne, France.

Mail: rodolphe.antoine@univ-lyon1.fr
}

\section{References}


[1] I. Chakraborty, T. Pradeep, Chemical Reviews 2017, 117, 8208.

[2] R. Jin, C. Zeng, M. Zhou, Y. Chen, Chemical Reviews 2016, 116, 10346.

[3] R. Antoine, V. Bonacic-Koutecky, Liganded silver and gold quantum clusters. Towards a new class of nonlinear optical nanomaterials, Springer, Cham, 2018.

[4] L.-Y. Chen, C.-W. Wang, Z. Yuan, H.-T. Chang, Analytical Chemistry 2015, 87, 216.

[5] P. Khandelwal, P. Poddar, Journal of Materials Chemistry B 2017, 5, 9055.

[6] X. Qu, Y. Li, L. Li, Y. Wang, J. Liang, J. Liang, Journal of Nanomaterials 2015, 2015, 23.

[7] Y. Lu, W. Chen, Analytical Chemistry 2015, 87, 10659.

[8] R. Hamouda, F. Bertorelle, D. Rayane, R. Antoine, M. Broyer, P. Dugourd, International Journal of Mass Spectrometry 2013, 335, 1.

[9] P. P. Radi, G. von Helden, M. T. Hsu, P. R. Kemper, M. T. Bowers, International Journal of Mass Spectrometry and Ion Processes 1991, 109, 49.

[10] M. F. Jarrold, J. E. Bower, The Journal of Chemical Physics 1993, 98, 2399.

[11] S. Gilb, P. Weis, F. Furche, R. Ahlrichs, M. M. Kappes, The Journal of Chemical Physics 2002, 116, 4094.

[12] F. Furche, R. Ahlrichs, P. Weis, C. Jacob, S. Gilb, T. Bierweiler, M. M. Kappes, The Journal of Chemical Physics 2002, 117, 6982.

[13] K. M. Harkness, L. S. Fenn, D. E. Cliffel, J. A. McLean, Analytical Chemistry 2010, 82, 3061.

[14] L. A. Angel, L. T. Majors, A. C. Dharmaratne, A. Dass, ACS Nano 2010, 4, 4691.

[15] M. R. Ligare, E. S. Baker, J. Laskin, G. E. Johnson, Chemical Communications 2017, 53, 7389.

[16] A. Baksi, A. Ghosh, S. K. Mudedla, P. Chakraborty, S. Bhat, B. Mondal, K. R.
Krishnadas, V. Subramanian, T. Pradeep, The Journal of Physical Chemistry $C$ 2017, 121, 13421.

[17] A. Baksi, S. R. Harvey, G. Natarajan, V. H. Wysocki, T. Pradeep, Chemical Communications 2016, 52, 3805.

[18] A. Soleilhac, F. Bertorelle, C. CombyZerbino, F. Chirot, N. Calin, P. Dugourd, R. Antoine, The Journal of Physical Chemistry C 2017, 121, 27733.

[19] S. Daly, C. M. Choi, A. Zavras, M. Krstić, F. Chirot, T. U. Connell, S. J. Williams, P. S. Donnelly, R. Antoine, A. Giuliani, V. Bonačić-Koutecký, P. Dugourd, R. A. J. O'Hair, The Journal of Physical Chemistry C 2017, 121, 10719.

[20] F. Bertorelle, I. Russier-Antoine, N. Calin, C. Comby-Zerbino, A. BensalahLedoux, S. Guy, P. Dugourd, P.-F. Brevet, Ž. Sanader, M. Krstić, V. BonačićKoutecký, R. Antoine, The Journal of Physical Chemistry Letters 2017, 8, 1979.

[21] A.-L. Simon, F. Chirot, C. M. Choi, C. Clavier, M. Barbaire, J. Maurelli, X. Dagany, L. MacAleese, P. Dugourd, Review of Scientific Instruments 2015, 86, 094101.

[22] H. E. Revercomb, E. A. Mason, Analytical Chemistry 1975, 47, 970.

[23] M. F. Mesleh, J. M. Hunter, A. A. Shvartsburg, G. C. Schatz, M. F. Jarrold, The Journal of Physical Chemistry 1996, 100, 16082.

[24] J. J. P. Stewart, Journal of Molecular Modeling 2013, 19, 1.

[25] M. James J. P. Stewart, Stewart Computational Chemistry, Colorado Springs, CO, USA, HTTP://OpenMOPAC.net, (2016).

[26] I. Russier-Antoine, F. Bertorelle, A. Kulesza, A. Soleilhac, A. BensalahLedoux, S. Guy, P. Dugourd, P.-F. Brevet, R. Antoine, Progress in Natural Science: Materials International 2016, 26, 455.

[27] C. Larriba-Andaluz, C. J. H. Jr., The Journal of Chemical Physics 2014, 141, 194107. 
[28] T. Wyttenbach, G. von Helden, J. J. Batka, D. Carlat, M. T. Bowers, Journal of the American Society for Mass Spectrometry 1997, 8, 275.

[29] G. Ben-Nissan, M. Sharon, Current Opinion in Chemical Biology 2018, 42, 25. 